\title{
Fabrication of a Microfluidic System Using Micromolded Alginate Gel as a Sacrificial Material for Tissues Engineering
}

\author{
Meng Wang $\mathbb{D},{ }^{1}$ Xiaoning Yang $\mathbb{D},{ }^{2}$ and Lanju Liang ${ }^{1}$ \\ ${ }^{1}$ School of Opto-Electronic Engineering, Zaozhuang University, Zaozhuang 277160, China \\ ${ }^{2}$ Research Institute of Surface Engineering, Taiyuan University of Technology, Taiyuan 030024, China
}

Correspondence should be addressed to Xiaoning Yang; yangxiaoning2007@163.com

Received 18 February 2020; Accepted 4 May 2020; Published 29 May 2020

Academic Editor: Thomas R. Caulfield

Copyright (c) 2020 Meng Wang et al. This is an open access article distributed under the Creative Commons Attribution License, which permits unrestricted use, distribution, and reproduction in any medium, provided the original work is properly cited.

We described a sacrificial molding for the formation of microfluidic networks. In this molding, the micromolded calcium alginate (Ca-Alg) is introduced as a sacrificial template. The basis of this procedure is fabricating a micromolded Ca-Alg hydrogel and encapsulating this model within a second gel and removing it by ion-exchange to leave a microchannel in the remaining gel. This microfluidic system can readily deliver solutes into the channels and even control the transport of solutes from channels into the bulk of the gels. Furthermore, the perfused vascular channels can sustain the metabolic activity of encapsulated cells, indicating the feasibility of this microfluidic system in the field of tissue engineering.

\section{Introduction}

The creation of three-dimensional (3D) vascularized tissues in vitro is highly desirable for potential clinical applications in tissue engineering [1-4]. In particular, in complex and thick organs, the vascularization of tissue structures is necessary not only to deliver nutrients and oxygen for the growth of cells but also to remove metabolic wastes in a biomimetic manner [5, 6]. In addition, vascularized tissues can accelerate functional anastomosis by forming natural connections with the host vasculature [7-9]. In general, vascularization is vital for the survival and functioning of implanted tissue-engineered constructs, as well as for ex vivo studies of vascular biology, cardiovascular pathology, drug delivery, and so on.

Microfluidic systems have emerged as a promising tool to develop the artificial microvasculature [10, 11]. The combination of hydrogels and microfabrication has further led to the development of microfluidic platforms, which offer precise control of the distribution of solute by utilizing the biomimetic convective and diffusive mass transfer [12]. The unique structure can deliver enough solutes to the interior of tissues to sustain the metabolism of embedded cells. Additionally, microfluidic networks can offer high- throughput capacity and simple convenient operation while defining microenvironmental conditions within tissues in health and pathology through spatiotemporal control. Therefore, microfluidic systems can not only facilitate vascularized networks in 3D-engineered tissue constructs but also provide biomimicking in vitro blood vessel models for biological research.

Recently, the 3D printing combining sacrificial molding technology has been paid great attention in the field of creating perusable channels within engineered tissues [13-15]. This strategy can be achieved by creating a 3D filament, casting the filament into a bulk material, and then sacrificing the filament to reveal a microfluidic architecture in the monolithic constructs. Miller et al. have used carbohydrate glass as a sacrificial template in engineered tissues to generate cylindrical networks. The carbohydrate glass can be dissolved easily in the aqueous solution [16]. Golden et al. constructed 3D cavities within collagen gels by heating and flushing the encapsulated micromolded gelatin [17].

Here, we propose the use of a calcium alginate $(\mathrm{Ca}-\mathrm{Alg})$ hydrogel as a sacrificial template for the formation of $3 \mathrm{D}$ cylindrical lumens. Alginates are a family of unbranched binary copolymers of 1-4 linked $\alpha$-L-guluronic acid (G) and $\beta$-D-mannuronic acid (M). Alginate gelation takes place 
when divalent cations (usually $\mathrm{Ca}^{2+}$ ) interact ionically with blocks of $\mathrm{G}$ residues resulting in a hydrogel $[18,19]$. Among the numerous merits of $\mathrm{Ca}-\mathrm{Alg}$, its sensitivity to chelating agents and ability to liquefy under mild conditions via ionexchange are attractive [20]. In recent years, calcium alginate (Ca-Alg) hydrogel has been mainly used as a tissue scaffold for cell culture and formation of functional tissue [21-23], but few studies have paid attention to its sacrificial properties in the field of microfluidic network.

\section{Results and Discussion}

2.1. Fabrication of the Alg-Sacrifice-Based Microfluidic Network. In this study, we intended to introduce calcium alginate hydrogel as a sacrificial template for the formation of microfluidic networks. The basis of this method is fabricating a micromolded calcium alginate hydrogel and encapsulating this model in a second gel and removing it by ion-exchange to leave microchannels in the remaining gel (Figure 1). The micromolded Ca-Alg hydrogel is firstly obtained by injecting $1.5 \%(w / v)$ sodium alginate solution into the $\mathrm{CaCl}_{2}$ solution $(100 \mathrm{mM})$ through a 32 -gauge steel nozzle and then immersed for up to $30 \mathrm{~min}$. The diameters of extruded $\mathrm{Ca}-\mathrm{Alg}$ can be controlled by varying the nozzle diameter and extrusion flow rate parameters. Furthermore, a suspension ECM prepolymer is poured to encapsulate the micromolded Ca-Alg.

In this study, agarose gels as the ECM are formed by mixing a low-melt agarose solution with cell suspension, dispensing the mixture around the micromolded Ca-Alg, and then placed at $4^{\circ} \mathrm{C}$ for $5 \mathrm{~min}$. After gelation of the ECM prepolymer, the micromolded $\mathrm{Ca}-\mathrm{Alg}$ is liquefied by the ionexchange effect of sodium citrate solution $(20 \mathrm{mg} / \mathrm{mL})$. As is shown in Figures 2(a)-2(c), the micromolded Ca-Alg within the ECM is liquefied and then removed by exhaustively flushing the gel through the channels. Basically, the gelation of alginate is a reversible process. The gelling of $\mathrm{Ca}-\mathrm{Alg}$ is initiated by the crosslinking of $\mathrm{Ca}^{2+}$ with $\mathrm{G}$ residues of alginate, resulting in an egg box structure at the molecular level. Inversely, the $\mathrm{Ca}-\mathrm{Alg}$ is fragile and easy to be liquefied by the ion-exchange of $\mathrm{Na}^{+}$in the solution (Figure 2(d)).

\subsection{Transport Characteristics of the Microfluidic Network.} To evaluate the transport properties of this modified microfluidic network, the delivery of two solutes including fluorescein (MW 376 Daltons) and fluorescein isothiocyanate-bovine serum albumin (FITC-BSA, MW 66,000 Daltons) is used. The distribution of solutes is created by injection of a pulse of fluorescein or FITC-BSA solution into the channels of microfluidic network. Fluorescence images for data were taken, and the transport extent was estimated by measuring the change in integrated fluorescence intensity with respect to time. To facilitate imaging of fluorescence distribution, we generated a single layer of fluidic channels formed within the center of a construct. The labeled solutes were introduced by the microflow pump into one end of the network and flushed out from the other end (Figure 3(a)). As shown in Figure 3, the microfluidic network formed in this study is highly diffusively permeable to both small molecules and macromolecules. Additionally, the transfer rate increased with a decrease in the molecular weight of the perfused mass (Figures 3(c) and 3(d)). The exchange of solutes within a bulk gel is generally conducted in two steps: convective mass transfer between the fluid and the walls of the microchannels and molecular diffusion between the walls and the bulk of the matrix (Figure 3(b)). The former is mainly determined by the construction of the microfluidic network and the flow rate, whereas the latter is primarily dependent on the molecular dimension and the structure of the matrix.

Furthermore, in order to selectively regulate the masstransport exchange within the matrix, we proposed to construct a functional membrane with selective permeability on the wall of microchannels (Figure 4(a)). Specifically, the functional membrane is formed by the crosslinking reaction between the micromolded $\mathrm{Ca}-\mathrm{Alg}$ and the chitosan. The transport properties of this modified microchannel are evaluated by injection of a pulse of small molecules and macromolecules into the channels. As shown in Figures 4(b) and 4(c), the fluorescein was readily delivered from channels into the surrounding gel within $3 \mathrm{~min}$ and subsequently transported to the bulk gel. The fast-diffused green fluorescence indicated the transport occurred by both convection and diffusion. In contrast to the small molecule, the diffusive motion of the FITC-BSA is hindered within the microchannels of the matrix due to the barrier effect of the formed membrane (Figures 4(d) and 4(e)). Therefore, the functional microchannels within this microfluidic network is endowed with a property of selective permeability, which can be used as a barrier model in the basic biological studies or pharmacological and toxicological screens.

\subsection{The Microfluidic Network Used in Tissue Engineering.} Human hepatocarcinoma HepG2 cells (Shanghai Institute of Biochemistry and Cell Biology) were chosen as a model to study the flexibility and compatibility of this microfluidic network. HepG2 cells $\left(5 \times 10^{5}\right.$ cells $\left./ \mathrm{mL}\right)$ were firstly suspended in the low-melt agarose $(2 \%, w / v)$. The suspension was gelled around the micromolded $\mathrm{Ca}-\mathrm{Alg}$ at $4^{\circ} \mathrm{C}$ for $5 \mathrm{~min}$ and then immersed in the solution of sodium citrate for $30 \mathrm{~min}$. After that, the dissolved alginate was flushed out by infusing PBS. By means of the microflow pump, the culture media was introduced to one end of the network at a flow rate of $10 \mu \mathrm{L} / \mathrm{min}$, allowing the media to perfuse the whole channels.

Compared to the cellular viability in the nonchanneled control gels, the encapsulated cells survived in the microfluidic scaffolds, indicating the biocompatibility of this network's forming process including removing the sacrificial Ca-Alg and casting the surrounding gels (Figures 5(a) and $5(\mathrm{~b})$ ). The survival of cells was mainly attributed to the rapid and biocompatible process. The time required for encapsulating cells and $\mathrm{Ca}-\mathrm{Alg}$ gels in ECM prepolymer, ECM crosslinking, and $\mathrm{Ca}-\mathrm{Alg}$ dissolution is within a few minutes. The sacrifice of Ca-Alg is developed by the ion-exchange of sodium citrate, which has no negative effects on cells. More importantly, the cellular activity in metabolically demanding 


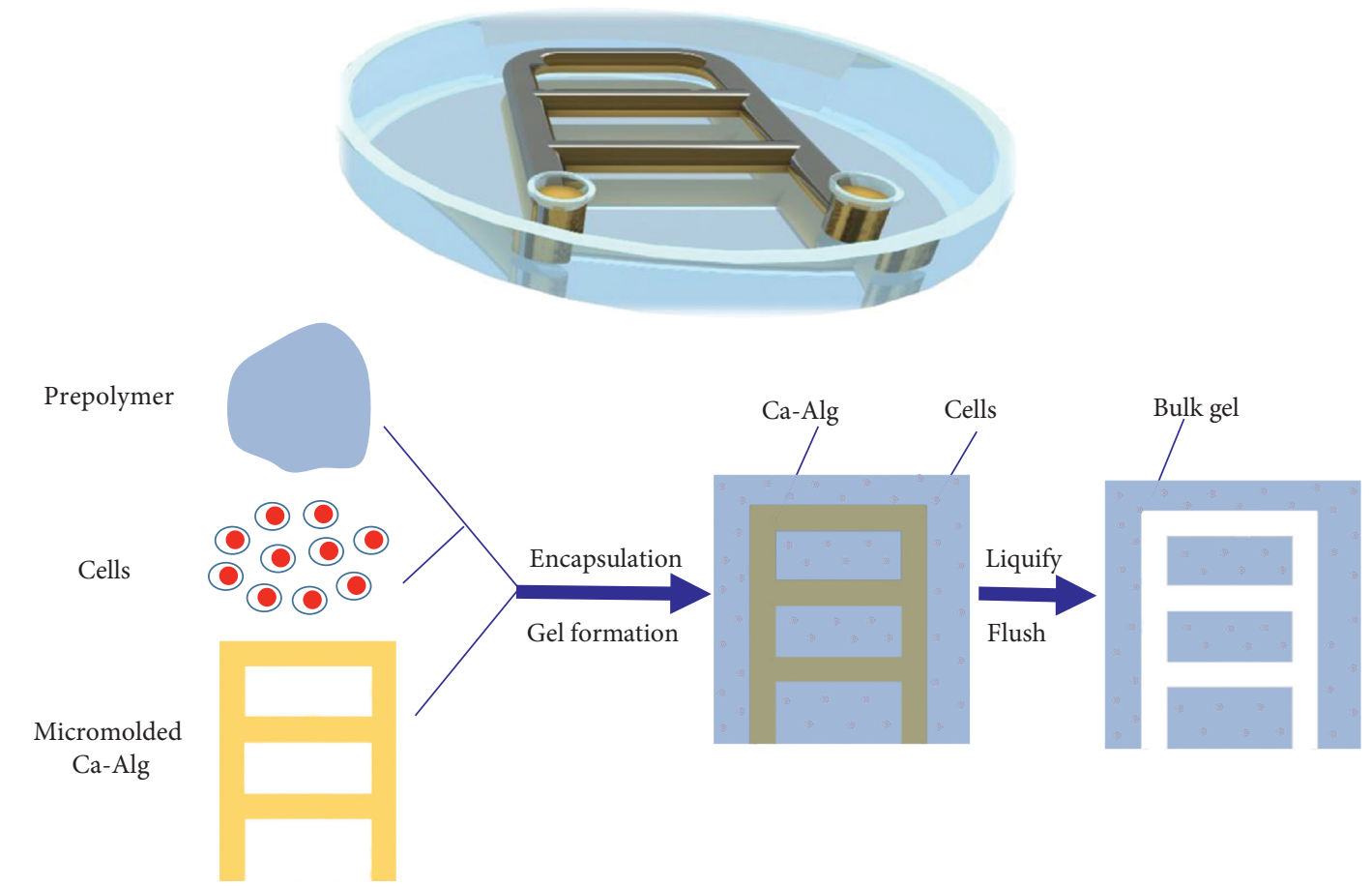

FIGURE 1: Schematic of the microfluidic network fabricated by sacrificing the micromolded Ca-Alg. The process includes fabricating a micromolded Ca-Alg, encapsulating this model in a second gel, and removing it by ion-exchange to leave microchannels in the remaining gel.

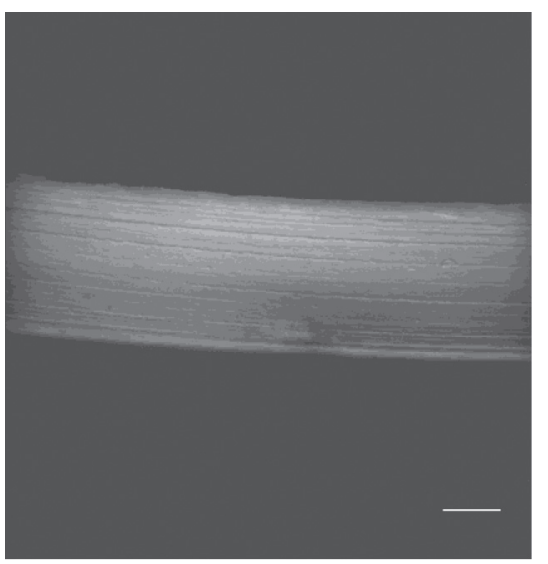

(a)
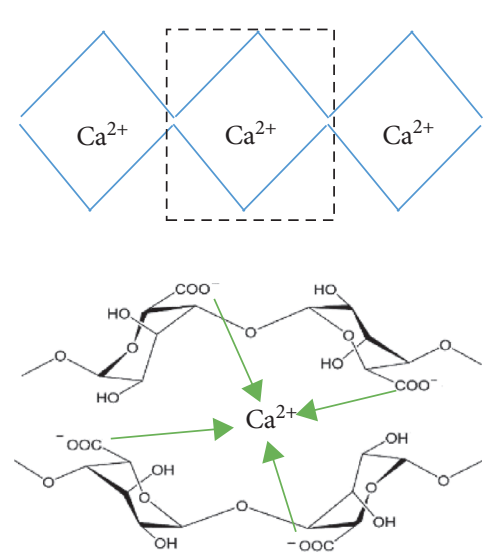

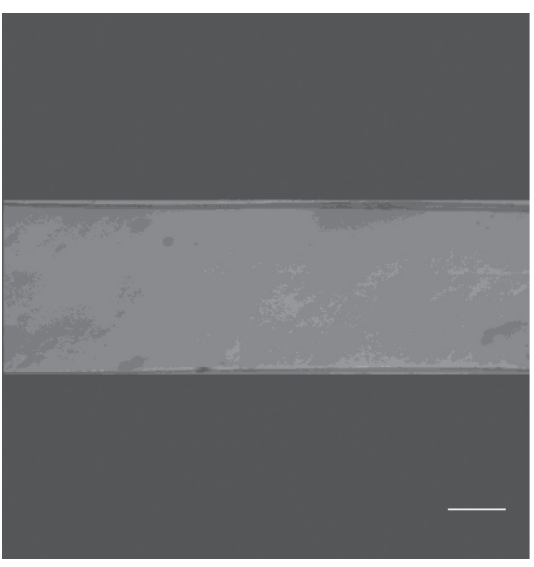

(b)

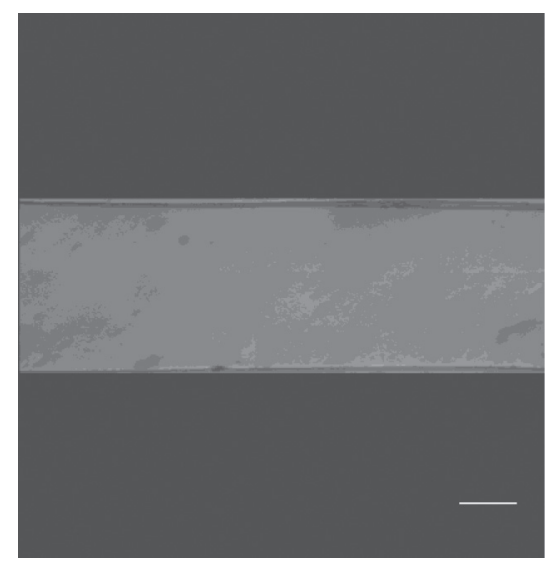

(c)
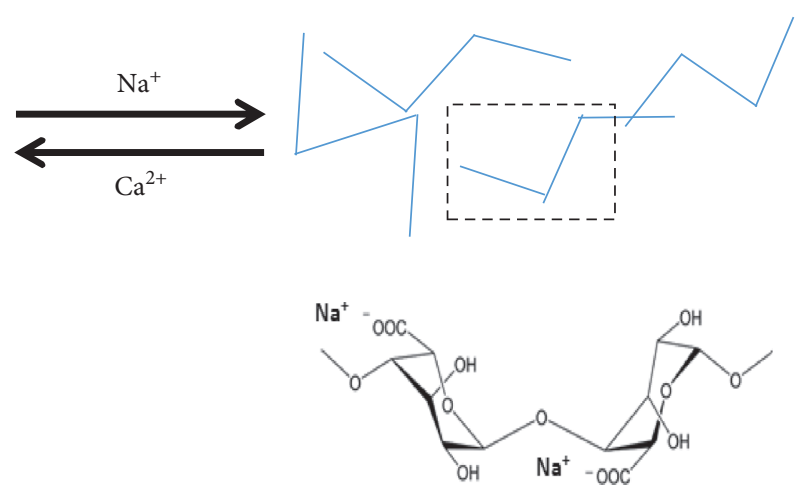

(d)

FIGURE 2: The forming process of microchannels recorded by the layer confocal microscopy. (a) The micromolded Ca-Alg is encapsulated within the bulk gel. (b) The Ca-Alg is liquefied. (c) The liquefied Ca-Alg is flushed out, and the microchannels are formed. (d) Schematic diagram of liquefying the Ca-Alg by the effect of ion-exchange. Bar: $200 \mu \mathrm{m}$. 


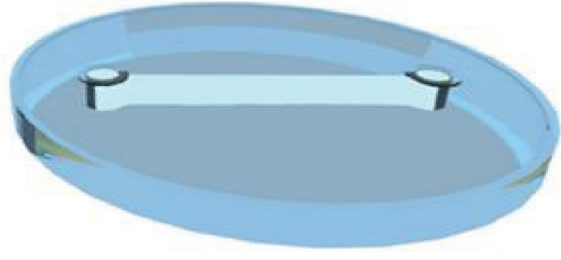

(a)

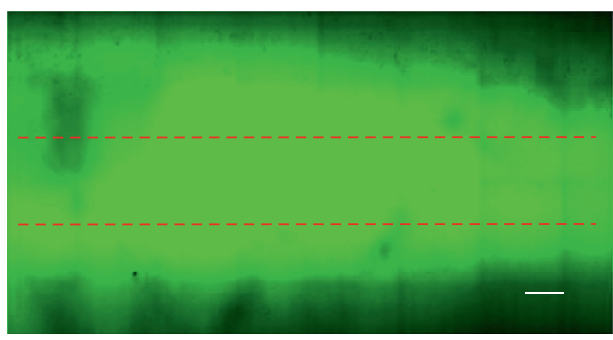

(c)

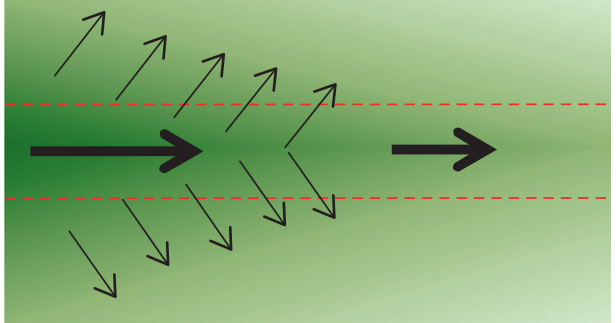

(b)

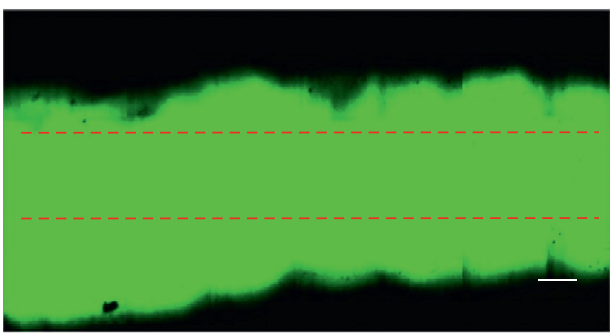

(d)

Figure 3: Transport of small molecule and macromolecule in the microfluidic network. (a) 3D schematic diagram of the microfluidic network. (b) The exchange of solutes within a bulk gel is generally conducted in two steps: convective mass transfer between the fluid and the walls of the microchannels and molecular diffusion between the walls and the bulk of the matrix. (c, d) Fluorescence distribution within the bulk matrix after injecting fluorescein and FITC-BSA solution into the channel for $10 \mathrm{~min}$, respectively. The red dotted line indicates the channel within the bulk matrix (bar: $200 \mu \mathrm{m}$ ).
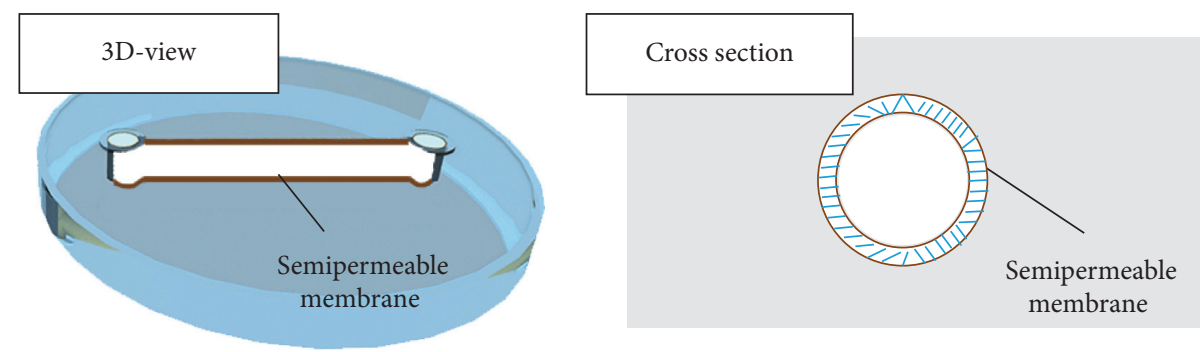

(a)
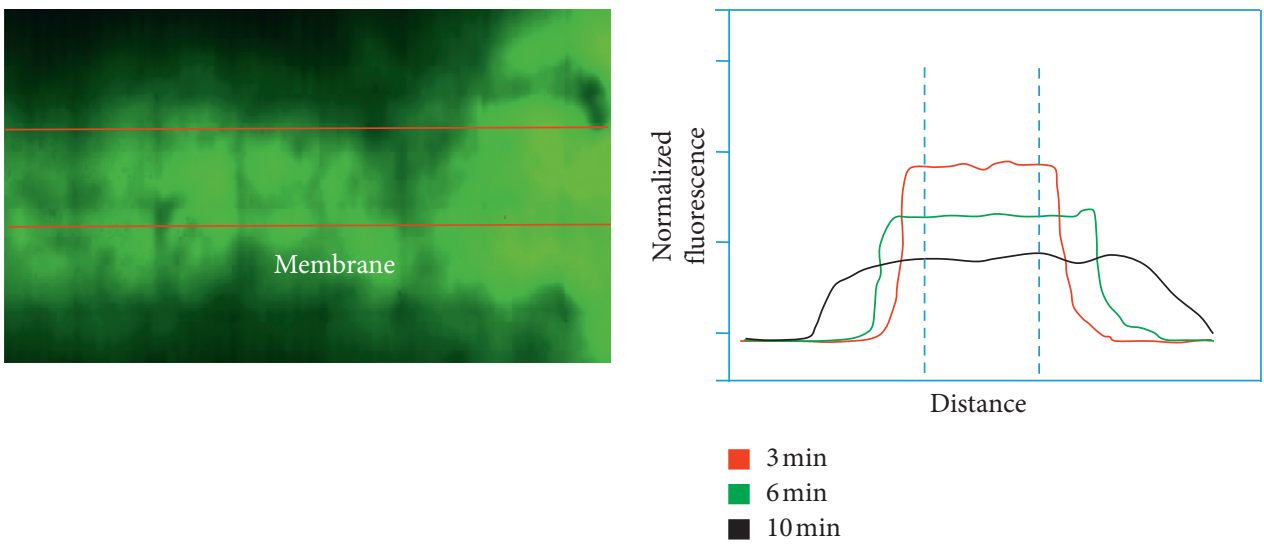

(b)

(c)

Figure 4: Continued. 


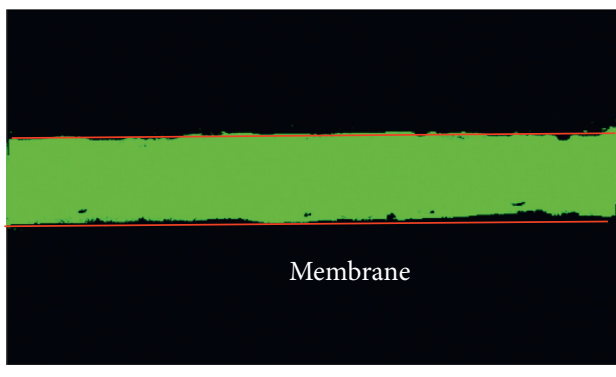

(d)

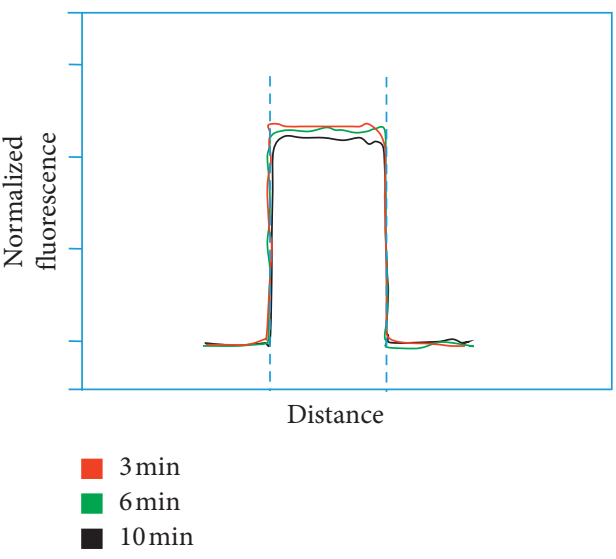

(e)

Figure 4: Transport of small molecule and macromolecule in the modified microfluidic network. (a) Schematic diagram of the modified microfluidic network shows that a membrane is constructed on the surface of channels. (b, d) Fluorescence distribution within the bulk matrix after injecting fluorescein and FITC-BSA solution into the channel for $10 \mathrm{~min}$, respectively. (c, e) Fluorescence intensity with respect to time after injecting fluorescein and FITC-BSA solution into the channel, respectively. The red full line indicates the modified channel within the bulk matrix (bar: $200 \mu \mathrm{m}$ ).
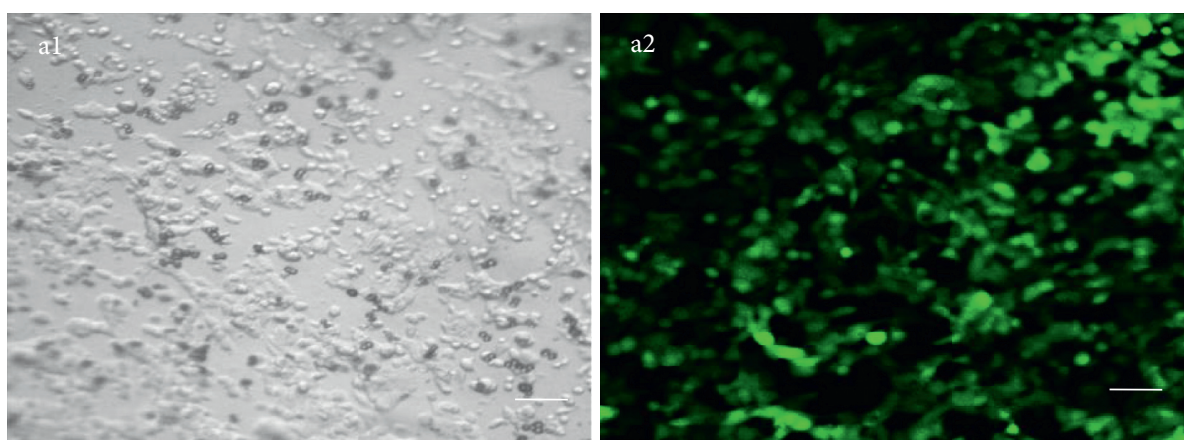

(a)
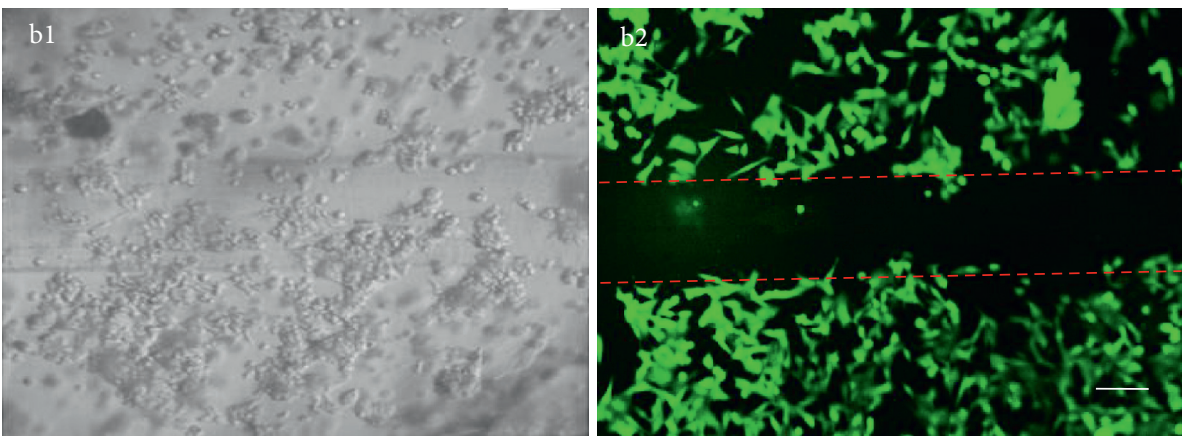

(b)

Figure 5: Continued. 

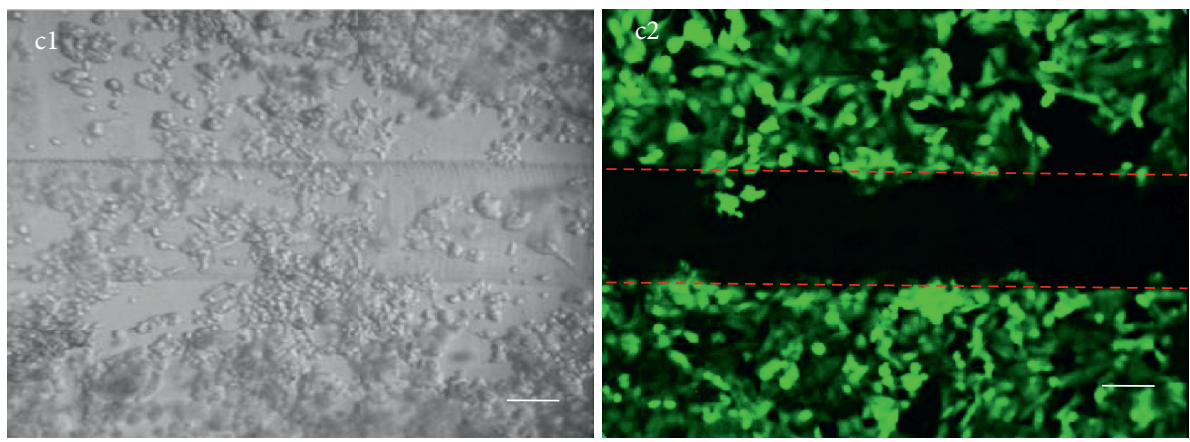

(c)

Figure 5: (a) Cells encapsulated within monolithic slab gels are considered as the control group. (b) Cells are encapsulated within the microfluidic network. (c) Cells encapsulated within the microfluidic network are cultured for 2 days. The cell morphology within the gel is shown by the microscopy (a1, b1, and c1). The live/dead staining of cells within the gel is shown by the laser confocal microscopy. Bar: $200 \mu \mathrm{m}$.

could be sustained by this microfluidic network. A necrotic core generally can be easily formed within the conventional 3D constructs densely populated with cells owing to lack of adequate mass transport. Nevertheless, few dead cells can be detected within the microfluidic scaffolds after perfused for 2 days (Figure 5(c)), demonstrating the feasibility of this microfluidic network in microfluidic devices and in tissue engineering.

In this study, we showed that the Ca-Alg was a suited sacrificial template for creating densely populated tissue constructs with perfusable microchannels. A key advantage of our approach is the convenient fabricating process, in which the microfluidic network is formed by encapsulating cells and $\mathrm{Ca}$-Alg gels in agarose and then by dissolving Ca-Alg. In theory, our approach may be used to form microchannels in any hydrogel. We believe that the microfluidic network prevents the formation of a necrotic core for highly metabolically demanding cells during in vitro $3 \mathrm{D}$ culture. Moreover, the microstructural characteristics of the microfluidic network such as vessel diameter, circularity, and surface modification can be regulated only by processing the Ca-Alg.

Vascularized tissues consists of three key compartments: the vascular lumen serving as the source of solutes for delivering, cells and matrix residing in the interstitial zone among vascular channels, and layer of endothelial cells lining the vascular wall. Here, we demonstrate control over the first two compartments in engineered tissue constructs. The formation of endothelium by seeding endothelial cells through the channels will be studied in our future work. In addition, although the Ca-Alg can dissolve biocompatibly and rapidly in the presence of living cells, the micromolded $\mathrm{Ca}-\mathrm{Alg}$ is too soft to physically support its own weight in an open 3D fabricating process. Thus, in order to expand the practicability of this approach, the mechanical stiffness of Ca-Alg needs to be stiffened by addition of some substances such as starch, glycerol, and dextran.

\section{Conclusion}

In summary, the approach described in this study provides a new opportunity to form a microfluidic network. This simple vascular forming approach allows independent control of the network geometry and extravascular constructs. Moreover, the formed microfluidic network in this study may construct a flexible platform for a wide array of specific applications, through regulating the soluble chemical environment within well-defined $3 \mathrm{D}$ cultures.

\section{Data Availability}

The data used to support the findings of this study are included within the article.

\section{Conflicts of Interest}

The authors declare that they have no conflicts of interest.

\section{Acknowledgments}

This project was supported by Qingchuang Science and Technology Plan of Shandong Universities (2019KJN001) and the National Natural Science Foundation of China (Grant nos. 61701434, 61704149, and 61675147).

\section{References}

[1] L. Liu and X. Wang, "Creation of a vascular system for organ manufacturing," International Journal of Bioprinting, vol. 1, no. 1, pp. 77-86, 2015.

[2] Y. Rui and J. T. Schantz, "In vitro angiogenesis of 3D tissueengineered adipose tissue," Journal of Bioactive and Compatible Polymers, vol. 24, no. 1, pp. 5-24, 2009.

[3] X. Wei, Y. Yan, R. Zhang et al., "Rapid prototyping of polyurethane for the creation of vascular systems," Journal of Bioactive and Compatible Polymers, vol. 23, no. 2, pp. 103-114, 2008.

[4] J. Fu and D. A. Wang, "In situ organ-specific vascularization in tissue engineering," Trends in Biotechnology, vol. 38, no. 8, 2018.

[5] A. F. Pellegata, A. M. Tedeschi, and D. C. Paolo, "Whole organ tissue vascularization: engineering the tree to develop the fruits," Frontiers in Bioengineering \& Biotechnology, vol. 6, no. 56, pp. 1-13, 2018. 
[6] P. Sofokleous, Functional 3D Ttissue Engineering Scaffolds Phase-Separation Technologies for 3D Scaffold Engineering, Elsevier, Amsterdam, Netherlands, 2018.

[7] C. Mazio, C. Casale, G. Imparato et al., "Prevascularized dermis model for fast and functional anastomosis with host vasculature," Biomaterials, vol. 192, pp. 159-170, 2018.

[8] W. Zhu, X. Qu, J. Zhu, X. Ma et al., "Direct 3D bioprinting of prevascularized tissue constructs with complex microarchitecture," Biomaterials, vol. 124, pp. 106-115, 2017.

[9] B.-N. B. Nguyen, R. A. Moriarty, T. Kamalitdinov, J. M. Etheridge, and J. P. Fisher, "Collagen hydrogel scaffold promotes mesenchymal stem cell and endothelial cell coculture for bone tissue engineering," Journal of Biomedical Materials Research Part A, vol. 105, no. 4, pp. 1123-1131, 2017.

[10] M. Fenech, V. Girod, V. Claveria, S. Meance, M. Abkarian, and B. Charlot, "Microfluidic blood vasculature replicas using backside lithography," Lab on a Chip, vol. 19, no. 5, pp. 2096-2106, 2019.

[11] Y. K. Hsieh, K.-P. Hsu, S.-K. Hsiao et al., "Laser-pattern induced contact guidance in biodegradable microfluidic channels for vasculature regeneration," Journal of Materials Chemistry $B$ Materials for Biology \& Medicine, vol. 6, no. 22, 2018.

[12] T. Takei, S. Sakai, and M. Yoshida, "In vitro formation of vascular-like networks using hydrogels," Journal of Bioscience and Bioengineering, vol. 122, no. 5, pp. 519-527, 2016.

[13] X. Zhao, L. Liu, J. Wang et al., "In vitro vascularization of a combined system based on a 3D printing technique," Journal of Tissue Engineering and Regenerative Medicine, vol. 10, no. 10, pp. 833-842, 2016.

[14] X. Wang, B. L. Rijff, and G. Khang, "A building-block approach to $3 \mathrm{D}$ printing a multichannel, organ-regenerative scaffold," Journal of Tissue Engineering and Regenerative Medicine, vol. 11, no. 5, pp. 1403-1411, 2017.

[15] X. Wang, Q. Ao, X. Tian et al., "Gelatin-based hydrogels for organ 3D bioprinting," Polymers, vol. 9, no. 9, pp. 1-24, 2017.

[16] J. S. Miller, K. R. Stevens, M. T. Yang et al., "Rapid casting of patterned vascular networks for perfusable engineered threedimensional tissues," Nature Materials, vol. 11, no. 9, pp. 768-774, 2012.

[17] A. P. Golden and J. Tien, "Fabrication of microfluidic hydrogels using molded gelatin as a sacrificial element," Lab on A Chip, vol. 7, no. 6, pp. 720-725, 2007.

[18] F. Wang, X. Lu, and X.-Y. Li, "Selective removals of heavy metals $\left(\mathrm{Pb}^{2+}, \mathrm{Cu}^{2+}\right.$, and $\left.\mathrm{Cd}^{2+}\right)$ from wastewater by gelation with alginate for effective metal recovery," Journal of Hazardous Materials, vol. 308, pp. 75-83, 2016.

[19] S. Benavides, P. Cortés, J. Parada, and W. Franco, "Development of alginate microspheres containing thyme essential oil using ionic gelation," Food Chemistry, vol. 204, pp. 77-83, 2016.

[20] University V., Low resource sample processor containing heat-activated surface tension valves, 2016.

[21] S. T. Bendtsen, S. P. Quinnell, and M. Wei, "Development of a novel alginate-polyvinyl alcohol-hydroxyapatite hydrogel for 3D bioprinting bone tissue engineered scaffolds," Journal of Biomedical Materials Research Part A, vol. 105, no. 5, pp. 1457-1468, 2017.

[22] G. Moumita, M. H. Sternfeld, I. Grinberg, and L. A. Abramovich, "Injectable alginate-peptide composite hydrogel as a scaffold for bone tissue regeneration," Nanomaterials, vol. 9, no. 4, pp. 1-15, 2019.

[23] T. Wang, Y. Zheng, Y. Shi, and L. Zhao, "pH-responsive calcium alginate hydrogel laden with protamine nanoparticles and hyaluronan oligosaccharide promotes diabetic wound healing by enhancing angiogenesis and antibacterial activity," Drug Delivery \& Translational Research, vol. 9, no. 1, 2018. 